\title{
Productividad de forraje de variedades de nopal (Opuntia spp.) bajo diferentes regímenes de humedad del suelo
}

\section{Forage productivity of nopal varieties (Opuntia spp.) under different soil moisture regimes}

Roberto Bacarrillo-López

Aurelio Pedroza-Sandoval ${ }^{2}$

Marco Antonio Inzunza Ibarra ${ }^{3}$ -

Arnoldo Flores-Hernández 2 [-

Francisco J. Macias-Rodriguez $[\mathbb{C}$

${ }^{1}$ Estudiante de Doctorado en Ciencias en Recursos Naturales y Medio Ambiente en Zonas Áridas de la Unidad Regional Universitaria de Zonas Áridas, UACh. Km. 38.5 Carretera Gómez Palacio - Chihuahua. CP. 56230. Bermejillo, Durango, México.

${ }^{2}$ Profesor-Investigador de la Unidad Regional Universitaria de Zonas Áridas de la Universidad Autónoma Chapingo. Km. 38.5 Carretera Gómez Palacio - Chihuahua. CP. 56230. Bermejillo, Durango, México.

${ }^{3}$ Centro de Investigación Disciplinaria en Relaciones Agua Suelo Planta Atmosfera del INFIFAP. Km. 6.5 margen derecha Canal de Sacramento, CP. 35140, Gómez Palacio, Durango, México.

${ }^{4}$ Profesor-Investigador del Centro Regional Universitario Centro Norte de la Universidad Autónoma Chapingo. Km. 24.5 Carretera Zacatecas-Fresnillo CP 98053, Morelos, Zacatecas, México.

*Autor de correspondencia:

apedroza@chapingo.uruza.edu.mx

\section{Artículo científico}

Recibido: 23 de febrero 2021

Aceptado: 25 de agosto 2021

Como citar: Bacarrillo-López R, Pedroza-Sandoval A, Inzunza Ibarra MA, Flores-Hernández A, MacíasRodríguez FJ (2021) Productividad de forraje de variedades de nopal (Opuntia spp.) bajo diferentes regímenes de humedad del suelo. Ecosistemas y Recursos Agropecuarios 8(3): e2878. DOI: 10.19136/era.a8n3.2878
RESUMEN. El objetivo de este estudio fue evaluar el crecimiento y producción de biomasa de variedades de nopal en diferentes contenidos de humedad del suelo como alternativa de producción forrajera. Se usó un diseño experimental de bloques al azar en un arreglo de parcelas divididas con tres repeticiones. Las parcelas grandes fueron los contenidos de humedad edáfica: muy favorable (22 - 27\%), favorable (16 - 21\%) y desfavorable (10 - 15\%); las parcelas chicas correspondieron a las variedades de nopal: Chapingo (Ch) Narro (N) y Escobar (E). La variedad Chapingo fue estadísticamente mayor $(P \leq 0.05)$ en ancho y grosor de cladodios en los contenidos de humedad del suelo favorable y muy favorable, con valores de 15.7 y $1.1 \mathrm{~cm}$ y 15.2 y $1.0 \mathrm{~cm}$, respectivamente; las variedades Escobar y Narro fueron las que registraron mayor contenido relativo de agua (CRA) en los contenidos de humedad favorable y desfavorable, con valores de 78.1 y $63 \%$, respectivamente. El índice de clorofila no varió por efecto de ninguno de los factores variación probados en este estudio. La variedad Narro fue la de mejor productividad en el contenido más favorable de humedad del suelo, con valores de $1.77 \mathrm{Kg}$ y $173.5 \mathrm{~g}$ de biomasa fresca y seca por planta, equivalente a 11.8 y $1.15 \mathrm{t} \mathrm{ha}^{-1}$ de biomasa fresca y seca, respectivamente, en corte de cladodios después de ocho meses de realizado el trasplante.

Palabras clave: Biomasa, cactáceas, deficiencia hídrica, sequía, zonas áridas.

ABSTRACT. The objective of this study was to evaluate the growth and biomass production of Opuntia varieties in different soil moisture content as an alternative for forage production. A randomized block experimental design was used in an arrangement of split plots with three replications. The large plots were the soil moisture content: very favorable (22 - 27\%), favorable (16 - 21\%) and unfavorable (10 - $15 \%)$; the small plots corresponded to the varieties of nopal: Chapingo (Ch) Narro (N) and Escobar (E). Chapingo variety was statistically higher $(P \leq 0.05)$ in width and thickness of cladodes in the favorable and very favorable soil moisture content, with values of 15.7 and $1.1 \mathrm{~cm}$ and 15.2 and 1.0 $\mathrm{cm}$, respectively; Escobar and Narro varieties were the ones that registered the highest relative water content (RWC) in the favorable and unfavorable moisture content, with values of 78.1 and $63 \%$, respectively. The chlorophyll index did not change due to the effect of any of the variation factors of this study. Narro variety was the one with the best productivity in the soil moisture content more favorable, with values of $1.77 \mathrm{~kg}$ and $173.5 \mathrm{~g}$ of fresh and dry biomass per plant, equivalent to 11.8 and $1.15 \mathrm{t} \mathrm{ha}^{-1}$ of fresh and dry biomass, respectively, in cladode cut after eight months after the transplant.

Key words: Biomass, cacti, water deficiency, drought, arid zones. 


\section{INTRODUCCIÓN}

Actualmente, el recurso agua presenta una situación de crisis ante condiciones de escasez por sequía, sobreexplotación del acuífero y contaminación con metales pesados y otros contaminantes (Azpilcueta-Pérez et al. 2018). A lo anterior, se añade el efecto del cambio climático, que influye sobre la disponibilidad hídrica con su impacto consecuente en la seguridad alimentaria (FAO 2013). En México, la sobre explotación de los acuíferos y la baja productividad del agua, hace urgente el diseño y aplicación de estrategias en el uso racional y eficiente de este recurso (CONAGUA 2010).

En la Comarca Lagunera, se ubica la principal cuenca lechera del país, con una explotación de 483 397 cabezas de ganado y una demanda diaria de 3 $732 \mathrm{t}$ de materia seca de forraje, principalmente alfalfa y gramíneas como maíz y sorgo (SIAP 2019). Para satisfacer estas necesidades, se han establecido los cultivos de alfalfa (Medicago sativa L.), maíz (Zea mays L.) y sorgo (Sorghum vulgare $\mathrm{H}$.), los cuales se cultivan en el ciclo de primavera-verano y, avena (Avena sativa L.), mezclas de trébol (Trifolium pratenese L.) y triticale ( $X$. Tríticosecale Wittmack) como forrajes de invierno (Pedroza et al. 2014). Sin embargo, la escaces y alto costo del agua de riego, la degradación de los suelos debido a problemas de salinidad y la poca diversidad de cultivos reducen la productividad y rentabilidad de los sistemas de producción agropecuarios (Montemayor et al. 2018).

El nopal es una cactácea con capacidad para crecer y desarrollarse de forma favorable ante condiciones ambientales limitantes propias de las zonas áridas, que lo convierte en perspectiva de producción en áreas agrícolas marginales. Es una planta suculenta de ruta fotosintética ácido crasuláceo que le permite adaptarse a condiciones de estrés hídrico, temperaturas extremas y salinidad, entre otros factores adversos (Torres-Ponce et al. 2015). Debido a este sistema fotosintético especializado y otras características como la suculencia de sus tejidos, la planta de nopal tiene una producción más eficiente de materia fresca y seca por unidad de agua consumida que supera a la que presentan los pastos y leguminosas y otras especies forrajeras (FAO-ICARDA 2018).

Durante los periodos de sequía, Opuntia con y sin espinas tiene un papel importante en el suministro de agua, fibra y nutrientes para la cría de animales bovinos, ovinos, caprinos y fauna silvestre (Feugang et al. 2006). Esta alternativa forrajera no es reciente, ya que el nopal ha desempeñado un rol importante para satisfacer la demanda de forraje suplementario en condiciones de sequía desde hace décadas (Torres-Ponce 2015). El objetivo de este estudio fue evaluar el crecimiento y producción de biomasa de variedades de nopal en diferentes contenidos de humedad del suelo como alternativa de producción de forraje en zonas áridas del norte de México.

\section{MATERIALES Y MÉTODOS}

\section{Ubicación del área de estudio}

El experimento se estableció en el campo experimental de la Unidad Regional Universitaria de Zonas Áridas (URUZA) de la Universidad Autónoma Chapingo (UACh) en Bermejillo, Durango, México. La región se ubica entre los $101^{\circ} 41^{\prime}$ y $104^{\circ} 61^{\prime}$ LO y $24^{\circ} 22^{\prime}$ y $26^{\circ} 23^{\prime} \mathrm{LN}$, a una altitud media de 1100 msnm. Presenta un clima seco desértico, con lluvias en verano e invierno fresco, la precipitación pluvial media anual es $258 \mathrm{~mm}$ y la evaporación potencial media anual de $2000 \mathrm{~mm}$, temperatura media anual de $21^{\circ} \mathrm{C}$ con máxima de $33.7{ }^{\circ} \mathrm{C}$ y mínima $7.5^{\circ} \mathrm{C}$ (Medina et al. 2005).

\section{Establecimiento del experimento}

El estudio se estableció en el ciclo otoño - verano del 2019 - 2020, en un área con suelo franco arcilloso. Las variedades de nopal con propósito de verdura y forraje provienen de la URUZA de la UACh en Bermejillo, Durango. México. Variedad Chapingo, cladodios grandes de color verde obscuro, forma circular o raqueta de 1 a $1.2 \mathrm{~cm}$ de grosor, sin espinas y flores de color rojo; Variedad Narro, cladodios grandes de color verde claro-oscuro, ligeramente alargados, agudos hacia su parte apical y planos, de 0.87 a $0.90 \mathrm{~cm}$ de grosor, ligera presencia de pequeñas espinas, flores de color amarillo; Variedad 
Escobar, cladodios grandes de color verde pálido, alargados, agudos hacia su parte apical y planos, de 0.82 a $0.84 \mathrm{~cm}$ de grosor, ligera presencia de pequeñas espinas hacia los bordes del cladodio, flores de color amarillo.

La plantación se realizó de forma manual, mediante excavación de una cepa de $30 \mathrm{~cm}$ de profundidad en suelo seco, colocando plantas de dos cladodios en doble hilera de $0.85 \mathrm{~m}$ de ancho entre hileras dentro de cama y $1.7 \mathrm{~m}$ entre camas a una distancia de $50 \mathrm{~cm}$ entre plantas con arreglo topológico 3 bolillo, para tener una densidad de población de 6667 plantas ha ${ }^{-1}$.

\section{Diseño experimental y de tratamientos}

Se utilizó un diseño experimental bloques al azar en un arreglo de parcelas divididas, con tres repeticiones. Las parcelas grandes fueron tres contenidos de humedad del suelo: muy favorable (22 - 27\%), favorable (16 - 21\%) y desfavorable (10 $15 \%)$, cuyos rangos fueron establecidos con base a la curva de abatimiento de humedad de Capacidad de Campo (CC) y Punto de Marchitez Permanente (PMP); las parcelas chicas fueron las variedades de nopal: Chapingo (Ch), Narro (N) y Escobar (E), con un total de nueve tratamientos producto de un factorial $3 \times 3$. La unidad experimental fue de 4 hileras de nopal de $8 \mathrm{~m}$ de longitud cada una, donde la parcela útil fueron las dos hileras centrales, a partir de las cuales se seleccionaron al azar cuatro plantas como tamaño de muestra para medir las variables respectivas.

\section{Contenido de humedad}

De acuerdo a la curva de abatimiento de humedad, determinada por el método de la olla de membrana (Richards 1948), la capacidad de campo (CC) del suelo del área experimental es de $25.5 \%$ de humedad edáfica; en tanto que el punto de marchitez permanente (PMP) fue de $13.1 \%$ (Figura 1). Las constantes de humedad de CC y PMP, fueron el referente para establecer los contenidos de humedad probados en este estudio (muy favorable, favorable y desfavorable), especificando que el nivel desfavorable (10-15\%) se llevó en su nivel mínimo, por debajo del PMP identificado, en la presunción de que la planta pudiera entrar a una condición de estrés, considerando que el nopal es una especie suculenta que evade el estrés hídrico a base de conservar apotenciales hídricos altos dentro de sus tejidos (Pedroza 1995).

Los tratamientos de los contenidos de humedad fueron dispuestos en las parcelas grandes, las cuales fueron colocadas en forma apareada, es decir un bloque consistió de tres parcelas grandes apareadas ubicadas horizontalmente y dentro de cada parcela grande se aleatorizaron las variedades de nopal. De esta manera se estableció una regadera principal con tuvo de PVC de 1" y a partir de éste se hicieron derivaciones con secciones horizontales con tuvo de PVC de 1/2" con llave de paso para controlar los tiempos de riego en cada contenido de humedad. Cada sección de éste último tubo con dos salidas conectadas a líneas regantes con goteros a distancia de $0.5 \mathrm{~m}$ entre ellos. Cada línea regante de un largo de $30 \mathrm{~m}$ lineales para regar dos hileras de plantas de nopal por cama, de acuerdo al arreglo topológico y tamaño de las unidades experimentales, las parcelas chicas de $8 \mathrm{~m}$ de longitud, $3 \mathrm{~m}$ de calle inicial a partir de la tuvería principal, $1 \mathrm{~m}$ de calle entre variedades de nopal y $1 \mathrm{~m}$ al final de la parcela grande.

Para determinar los tiempos de riego, se consideraron diferentes características físico-químicas del suelo, tales como: densidad aparente de $1.2 \mathrm{~g}$ $\mathrm{cm}^{-3}$, capilaridad de $\mathrm{L}=2.3669 \mathrm{~T} 0.4215(\mathrm{~L}$ en $\mathrm{cm}$ y $T$ en min), velocidad de infiltración de $1.3 \mathrm{~cm} \cdot \mathrm{h}^{-1}$, evapotranspiración media diaria de $11 \mathrm{~mm}$ y coeficiente experimental de evaporación de $70 \%$. El cálculo del tiempo de riego se hizo conforme a la metodología citada por Pedroza y Durán (2005) y mediante el suministro controlado de agua de acuerdo a lo reportado por Jiménez-Galindo y Acosta-Gallegos (2013).

Para medir el contenido de humedad, se monitoreó constantemente el suelo con un medidor digital (Soil Tester modelo HB-2) con lectura en tiempo real. Para ello, después del riego inicial estandarizado a capacidad de campo, se dejó abatir la humedad edáfica de acuerdo con los tratamientos de humedad del suelo: muy favorable (22-27\%), favorable (16-21\%) y 


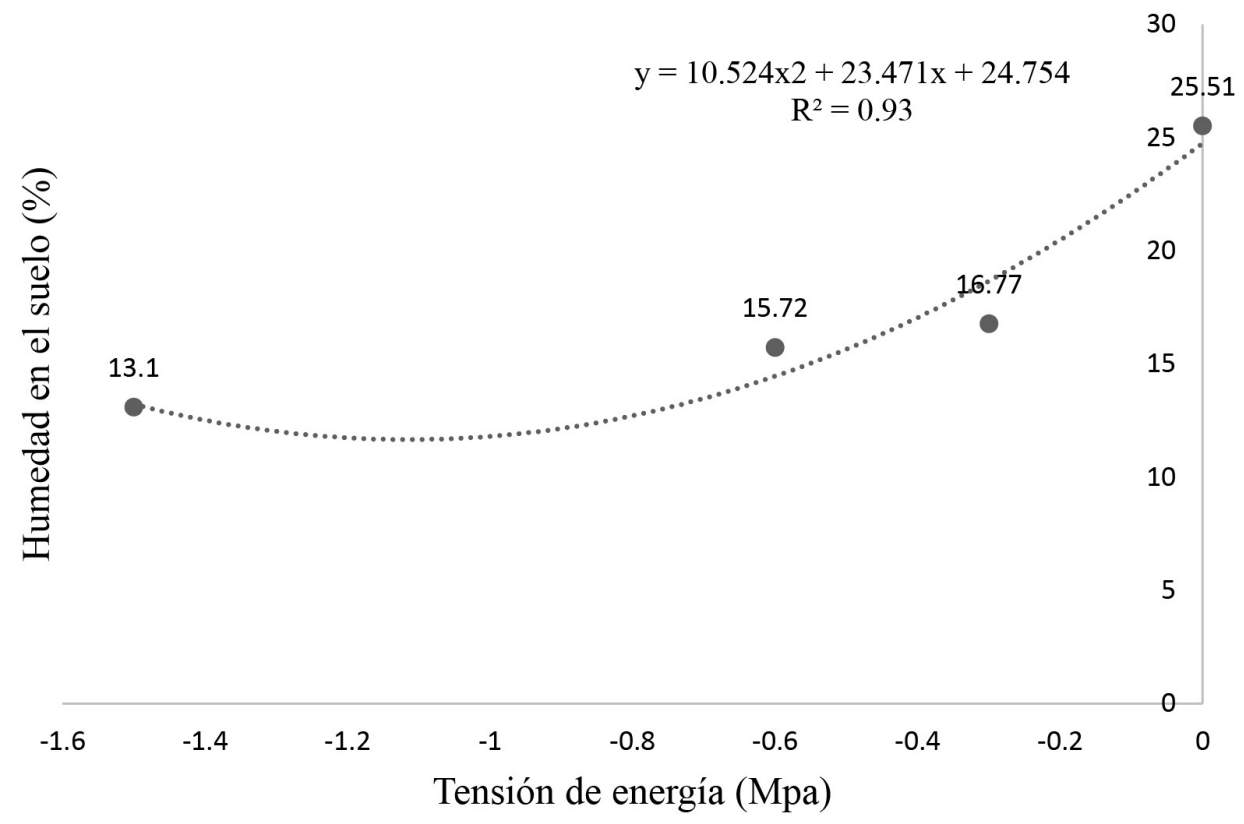

Figura 1. Curva de retención de humedad del suelo del área experimental.

desfavorable (10-15\%), procediendo a los riegos de recuperación cuando se detectó un abatimiento de $5 \%$ en cada uno de los tres niveles de humedad del suelo.

\section{Variables respuesta}

Las variables de crecimiento y desarrlo de planta y cladodios se midieron durante un período de 240 días después del trasplante (DDP), correspondiente a un períodos aproximado de ocho meses en otoño-verano de 2019-2020. Variables de crecimiento de planta y cladodios: altura de planta $(\mathrm{cm})$ y crecimiento de cladodios, éstos últimos referido a longitud $(\mathrm{cm})$, ancho $(\mathrm{cm})$ y grosor $(\mathrm{cm})$, con uso flexómetro y vernier, ambos marca Truper. Variables fisiológicas: contenido relativo de agua (CRA) (\%), determinado de acuerdo a la metodología reportada por Kramer (1983), donde se aplicaron las siguientes ecuaciones: $C R A=\frac{\text { Peso fresco }- \text { Peso seco }}{\text { Peso turgente }- \text { Peso seco }} \times 100, D H=$ $\frac{\text { Peso turgente }- \text { Peso seco }}{\text { Peso fresco }- \text { Peso seco }} x-100$ y $C R A=100-D H$. Dónde: CRA: contenido relativo de agua; $\mathrm{DH}$ : déficit hídrico; índice de clorofila, medida con uso de determinador de clorofila Marca Spectrum Technologies
Inc. Fieldscout CM 1000.

Las variables de productividad correspondieron a: número de cladodios por planta, peso fresco y seco de biomasa (g) por planta, las dos últimas determinadas mediante uso de balanza granataria. Para la determinación del peso de biomasa fresco y seco se cortaron cuatro cladodios de cada planta, posteriormente el promedio de estos valores, fue multiplicado por el número total de cladodios por planta. Para el peso seco, los cladodios fueron cortados en línea recta a todo lo largo del borde, posteriormente fueron secados en una estufa a $65{ }^{\circ} \mathrm{C}$ por un período de tiempo hasta llegar a peso constante. Con base en ello se estimó la producción de forraje fresco y seco por hectárea de acuerdo a la densidad de plantación usada en el experimento.

\section{Análisis de datos}

Con la base de datos se realizaron análisis de varianza y prueba de rango múltiple de medias Tukey para identificar el efecto de tratamiento, mediante el programa estadístico Minitab 16 y SAS versión 9.0. Para el análisis de regresión se usó el Programa Excel 2016. 


\section{RESULTADOS}

\section{Crecimiento de planta y cladodios}

El ancho y grosor del cladodio no variaron entre variedades en el contenido de humedad desfavorable (10-15\%), pero en los contenidos de humedad muy favorable (22-27\%) y favorable (16$21 \%)$, la variedad Chapingo fue significativamente mayor $(\mathrm{P} \leq 0.05)$, con valores de 15.7 y $1.1 \mathrm{~cm}$ y 15.2 y $1.0 \mathrm{~cm}$, respectivamente. Las variedades Escobar y Narro, fueron estadísticamente menores para estas dos variables y sin diferencia estadística entre ambas. La longitud de cladodio (Tabla 1) y altura de la planta (Figura 2), no mostraron ningún efecto por variedad en los diferentes contenidos de humedad del suelo probados en este estudio.

\section{Contenido relativo de agua y clorofila}

El contenido relativo de agua (CRA) tuvo un comportamiento inverso respecto al de crecimiento de los cladodios, ya que fue significativamente más bajo en la variedad Chapingo en los contenidos favorable y desfavorable de humedad del suelo, con valores de 68.1 y $36.6 \%$, respectivamente, respecto de la variedad Escobar que registró lo mayores valores con 78.1 y $63 \%$, respectivamente; y fue estadísticamente igual a la variedad Narro. No hubo diferencias significativas en el índice de clorofila en las tres variedades dentro de cada contenido de humedad del suelo (Tabla 2).

\section{Productividad}

La variedad Escobar fue significativamente mayor en el número de cladodios por planta (NCP) con valores de $8,6.7$ y 5.2 en los contenidos muy favorable, favorable y desfavorable de humedad edáfica, respectivamente; la variedad Chapingo fue la de menor efecto en esta variable con valores de 6, 4 y 2.8, respectivamente. La variedad Narro, mostró un comportamiento de tendencia intermedia con respecto a las otras dos variedades en los contenidos favorable y desfavorable de humedad en el suelo (Tabla 3).

La variedad Narro fue la de mejor respuesta en producción de forraje en términos de peso fresco y seco de biomasa por planta, medidos ocho meses después del trasplante, con respecto a las variedades Escobar y Chapingo, con valores de 1.77, 1.52 y 1.26 $\mathrm{kg}$ de peso fresco y de 173.5, 149.7 y $124.5 \mathrm{~g}$ de peso seco, respectivamente; la variedad Escobar tuvo una respuesta intermedia entre las variedades Narro y Chapingo, ésta última de comportamiento más pobre (Tabla 3).

\section{DISCUSIÓN}

La no variación en el ancho y grosor del cladodio por efecto de variedad de nopal en contenido desfavorable de humedad en el suelo, confirma la tolerancia al estrés hídrico de esta cactácea, asociada a la capacidad de conservar potenciales hídricos en sus tejidos hasta determinados límites de estrés por falta de agua en el ambiente, particularmente el suelo (Pedroza 1995). Lo anterior sugiere que, en casos de déficit hídrico por falta de suficiente disponibilidad de agua, cualquiera de las tres variedades puede ser utilizada sin presentar impactos negativos en el crecimiento de cladodio, que es la unidad básica de producción de forraje. En cambio, cuando hay disponibilidad hídrica en condición favorable a muy favorable, la mejor opción es la variedad Chapingo, en términos de mejor ancho y grosor del cladodio.

Esta respuesta de mecanismo de tolerancia por evasión mediante la capacidad de conservar potenciales hídricos en los tejiidos en condiciones de sequía, puede variar dependiendo de la etapa fenológica de la planta y el cladodio, así como del material genético utilizado, entre otros factores (Becerril y Peña 2006). Al respecto, Alessio et al. (2016) en un estudio con plantas de Opuntia en macetas sin riego, reportan que la sequía disminuyó el grosor del cladodio y el crecimiento del mismo, observando que los cladodios más jóvenes perdieron agua más tardíamente que los más viejos. Mientras que LópezCollado et al. (2013), encontraron diferencias estadísticas $(p \leq 0.05)$ entre variedades en longitud, ancho y grosor de brotes de cladodios. Aun cuando para la longitud del cladodio no hubo efecto por variedad ni contenido de humedad en el suelo, esta variable fue 
Tabla 1. Efecto en el crecimiento de cladodios a tres contenidos de humedad del suelo en diferentes variedades de nopal (Opuntia spp) 240 DDT.

\begin{tabular}{|c|c|c|c|c|c|c|c|c|c|}
\hline \multirow[t]{3}{*}{ Variedad de nopal } & \multicolumn{9}{|c|}{ Contenido de humedad en el suelo (\%) } \\
\hline & \multicolumn{3}{|c|}{$\begin{array}{c}\text { Muy favorable } \\
(22-27)\end{array}$} & \multicolumn{3}{|c|}{$\begin{array}{c}\text { Favorable } \\
(16-21)\end{array}$} & \multicolumn{3}{|c|}{$\begin{array}{c}\text { Desfavorable } \\
(10-15)\end{array}$} \\
\hline & $\begin{array}{l}\mathrm{LC} \\
(\mathrm{cm})\end{array}$ & $\begin{array}{l}\mathrm{AC} \\
(\mathrm{cm})\end{array}$ & $\begin{array}{l}\mathrm{GC} \\
(\mathrm{cm})\end{array}$ & $\begin{array}{l}\mathrm{LC} \\
(\mathrm{cm})\end{array}$ & $\begin{array}{l}\mathrm{AC} \\
(\mathrm{cm})\end{array}$ & $\begin{array}{l}\mathrm{GC} \\
(\mathrm{cm})\end{array}$ & $\begin{array}{l}\mathrm{LC} \\
(\mathrm{cm})\end{array}$ & $\begin{array}{l}\mathrm{AC} \\
(\mathrm{cm})\end{array}$ & $\begin{array}{l}\mathrm{GC} \\
(\mathrm{cm})\end{array}$ \\
\hline Chapingo & $27.2^{a}$ & $15.7^{a}$ & $1.1^{a}$ & $23.8^{a}$ & $15.2^{a}$ & $1.0^{a}$ & $16.8^{a}$ & $10.5^{a}$ & $0.66^{a}$ \\
\hline H. Escobar & $28.5^{a}$ & $14.3^{a b}$ & $0.89^{b}$ & $24.2^{a}$ & $12.5^{b}$ & $0.81^{b}$ & $17.5^{a}$ & $9.3^{a}$ & $0.55^{a}$ \\
\hline Narro & $27.8^{a}$ & $13.5^{b}$ & $0.83^{b}$ & $25.2^{a}$ & $12.9^{b}$ & $0.85^{b}$ & $18.4^{a}$ & $9.9^{a}$ & $0.53^{a}$ \\
\hline Promedio & 27.8 & 14.5 & 0.94 & 24.4 & 13.5 & 0.88 & 17.5 & 9.9 & 0.58 \\
\hline
\end{tabular}



Figura 2. Comportamiento de la altura de planta en tres variedades de nopal (Opuntia spp) en diferentes contenidos de humedad del suelo. Prueba de Tukey $(P \leq 0.05)$. Cifras con las mismas letras dentro de cada contenido de humedad del suelo, son estadísticamente iguales.

Tabla 2. Contenido relativo de agua en tres variedades de nopal (Opuntia spp) en diferentes contenidos de humedad en el suelo.

\begin{tabular}{|c|c|c|c|c|c|c|}
\hline \multirow{3}{*}{ Variedad de nopal } & \multicolumn{6}{|c|}{ Contenido de humedad en el suelo (\%) } \\
\hline & \multicolumn{2}{|c|}{$\begin{array}{c}\text { Muy favorable } \\
(22-27)\end{array}$} & \multicolumn{2}{|c|}{$\begin{array}{c}\text { Favorable } \\
(16-21)\end{array}$} & \multicolumn{2}{|c|}{$\begin{array}{c}\text { Desfavorable } \\
(10-15)\end{array}$} \\
\hline & CRA (\%) & CLOR & CRA (\%) & CLOR & CRA (\%) & CLOR \\
\hline Chapingo & $77.9^{a}$ & $142.2^{a}$ & $68.1^{b}$ & $144.5^{a}$ & $36.6^{b}$ & $147.5^{a}$ \\
\hline Escobar & $82.8^{a}$ & $143.4^{a}$ & $78.1^{a}$ & $144.4^{a}$ & $63.0^{a}$ & $147.5^{a}$ \\
\hline Narro & $77.5^{a}$ & $144.3^{a}$ & $77.9^{a}$ & $144.0^{a}$ & $62.6^{a}$ & $147.0^{a}$ \\
\hline
\end{tabular}

estadísticamente iguales. $\mathrm{CRA}=$ Contenido relativo de Agua, $\mathrm{CLOR}=$ Clorofila .

mayor que lo reportado por Sánchez et al. (2018) y por Ruiz-Espinoza et al. (2008), con valores de 10.76 a $11.55 \mathrm{~cm}$ y promedio de $20 \mathrm{~cm}$, respectivamente.
En este estudio, la longitud de cladodio, dependió del contenido de humedad en el suelo, con valores de $27.2,23.8$ y $17.5 \mathrm{~cm}$ en contenidos de humedad 
Tabla 3. Efecto del contenido de humedad del suelo en tres variedades de nopal (Opuntia spp) en la productividad de biomasa fresca y seca a los 240 DDT.

\begin{tabular}{|c|c|c|c|c|c|c|c|c|c|}
\hline \multirow[t]{3}{*}{ Variedad de nopal } & \multicolumn{9}{|c|}{ Contenido de humedad en el suelo (\%) } \\
\hline & \multicolumn{3}{|c|}{$\begin{array}{c}\text { Muy favorable } \\
(22-27)\end{array}$} & \multicolumn{3}{|c|}{$\begin{array}{c}\text { Favorable } \\
(16-21)\end{array}$} & \multicolumn{3}{|c|}{$\begin{array}{c}\text { Desfavorable } \\
(10-15)\end{array}$} \\
\hline & $\begin{array}{c}\text { PFBP } \\
(\mathrm{kg})\end{array}$ & $\begin{array}{l}\text { PFBP } \\
\text { (g) }\end{array}$ & NCP & $\begin{array}{l}\text { PSBP } \\
(\mathrm{kg})\end{array}$ & $\begin{array}{l}\text { PFBP } \\
\text { (g) }\end{array}$ & NCP & $\begin{array}{l}\text { PSBP } \\
(\mathrm{kg})\end{array}$ & $\begin{array}{c}\text { PSBP } \\
\text { (g) }\end{array}$ & NCP \\
\hline Chapingo & $1.26^{b}$ & $124.5^{b}$ & $6.0^{b}$ & $1.06^{a}$ & $105.6^{a}$ & $4.0^{b}$ & $0.44^{a}$ & $44.1^{a}$ & $2.8^{b}$ \\
\hline Escobar & $1.52^{a b}$ & $149.7^{a b}$ & $8.0^{a}$ & $1.28^{a}$ & $127.9^{a}$ & $6.7^{a}$ & $0.38^{a}$ & $37.7^{a}$ & $5.2^{a}$ \\
\hline Narro & $1.77^{a}$ & $173.5^{a}$ & $8.3^{a}$ & $1.05^{a}$ & $104.2^{a}$ & $5.9^{a b}$ & $0.44^{a}$ & $43.6^{a}$ & $4.2^{a b}$ \\
\hline Promedio & 1.51 & 149.2 & 7.4 & 1.13 & 112.5 & 5.5 & 0.42 & 41.8 & 4.0 \\
\hline
\end{tabular}

muy favorable, favorable y desfavorable, respectivamente. La altura de planta fue menor (39.5 a 67.6 $\mathrm{cm})$ a lo reportado por Ferreira et al. (2003) quienes encontraron valores entre $70-122 \mathrm{~cm}$ en nopal forrajero Opuntia fícus-indica Mill, lo cual puede estar relacionado con las diferencias del material genético utilizado y del tiempo de corte de los cladodios, el cual fue de 12 meses en lo reportado por estos autores y de ocho meses en el presente estudio.

El contenido relativo de agua (CRA), referido al contenido hídrico de las plantas ante condiciones de estrés por deficiencia hídríca, es de vital importancia para la sobrevivencia y la productividad. EI CRA, es el indicador más común para medir el estado hídrico de los tejidos en la planta, es la medida del contenido de agua respecto del total que los tejidos pueden almacenar y se expresa en porcentaje. Se relaciona con el potencial hídrico, ya que éste y sus componentes, potencial de presión y potencial osmótico, están en función del volumen de agua en el protoplasma (Argentel et al. 2006). De acuerdo a lo anterior, las variedades Escobar y Narro, son las que tienen la mayor tolerancia al estrés moderado y alto mediante la capacidad de retención de agua en sus tejidos, lo cual sugiere una mejor respuesta de comportamiento productivo ante condiciones de déficit hídrico en el suelo. En tanto que la variedad Chapingo, se puede considerar como la de menor capacidad de retención de agua en sus tejidos ante contenidos medios y bajos de humedad del suelo, afectando en estas condiciones el desarrollo y crecimiento del cladodio; pero de mejor respuesta en estas variables ante condiciones muy favorables de humedad edáfica. Al respecto, se indi- can que los materiales genéticos que no evaden las sequía sino que más bien la toleran, son aquéllos que tienen mayor capacidad de soportar potenciales hídricos bajos en sus tejidos, aunque ello puede repercutir en un detrimento de productividad (Aranda et al. 2014).

Con respecto al efecto de tratamiento en el contenido de clorofila, diversos autores indican que el contenido de los pigmenteos fotosintéticos depende de una gran diversidad de factores ambienteales y de la propia planta, como la temperatura, radiación solar, contenido hídrico, etapa fenológica de la planta, tipo de especie, entre otros (Casierra-Posada et al. 2013). Al respecto, Becerril y Peña (2006) reportan que la sequía redujo la fluorescencia de la clorofila tanto en el clorénquima como en el parénquima en Opuntia ficus-indica. Mientras que Meléndez et al. (2006) y Trujillo et al. (2010), reportaron que el contenido de clorofila aumenta y disminuye en suelos con bajos y altos gradientes de humedad del suelo, respectivamente. Sobre lo mismo Aguilar y Peña (2006), en un estudio realizado en Opuntia ficus-indica, encontraron que las plantas bajo sequía severa (sin ningún riego) redujeron significativamente la concentración de clorofilas; en el clorénquima, la clorofila $a+b$ disminuyó en $42.3 \%$, la clorofila a en $34.2 \%$ y la clorofila b en $31.4 \%$; en el parénquima, los decrementos de clorofila $a+b$, a y b fueron de $39.6 \%, 35.8 \%$ y $23.6 \%$, respectivamente. Con base en estos antecedentes, la ausencia de efecto del contenido de humedad en el contenido de clorofila del presente estudio en ninguna de las variedades, pudiera estar relacionado a que el contenido más bajo de humedad (10-15\%) no fue su- 
ficiente para generar una condición de estrés a nivel celular que incidiera en la degradación de este compuesto fotosintético.

El efecto observado en la variedad Chapingo, con respecto a tener un significativo menor CRA en contenidos muy favorables y favorables de humedad edáfica y aún así tener un mejor ancho y grosor de hoja, significa su capacidad de evadir la deficiencia de agua en el suelo, a base de mantener potenciales hídricos moderados que induzcan un adecuado desarrollo y crecimiento de los cladodios, pero no así su capacidad reproductiva en término del número de cladodios por planta, el cual es menor, respecto a las variedades Escobar y Narro. Al respecto, Florido y Bao (2014) reportan que el estrés hídrico a nivel de sobrevivencia, tiene como repercusión una menor capacidad reproductiva en las plantas. Al respecto, López-Collado (2013) reportó que el material genético de nopal identificado con clave NDV fue el que presentó mayor número de brotes con un valor de 43.5, respecto de otros materiales genéticos evaluados. La producción de brotes de cladodios del presente estudio, es menor a los reportados por RuizEspinoza et al. (2008) y por Sánchez et al. (2018), quienes reportan entre 10.7 a 11.15 brotes de cladodios por penca madre, respectivamente, en tanto que en el presente estudio fue entre 4.0 y 7.4. La biomasa fresca, es la variable más directamente relacionada con la productividad de forraje, y la biomasa seca tiene potencial en las industrias alimenticia y farmacéutica (SAGARPA 2015). Ambas variables, peso fresco y seco de biomasa, fueron significativamente mayores en las variedades Escobar y Narro, principalmente esta última, en condiciones de contenido hídrico muy favorable. Este efecto de respuesta, está asociado a la capacidad reproductiva en número de cladodios por planta.

De acuerdo con la densidad de plantas de 6 667 plantas $\mathrm{h}^{-1}$, se estimó un rendimiento de 8.4, 10.1 y $11.8 \mathrm{t} \mathrm{ha}^{-1}$ de biomasa fresca y de $0.83,0.998$ y $1.156 \mathrm{t} \mathrm{ha}^{-1}$ de biomasa seca en corte de cladodios a los ocho meses de realizado el trasplante de las variedades Chapingo, Escobar y Narro, respectivamente. Estos valores de producción por unidad de superficie son superiores a los encontrados por Sánchez et al. (2018), quienes reportaron entre 0.347 a $0.447 \mathrm{t} \mathrm{ha}^{-1}$ de biomasa seca, bajo diferentes densidades de población en Opuntia ficus-indica. En tanto que Flores-Hernández et al. (2019), reportaron productividad de biomasa fresca de nopal entre 106.4 y 199.5 t ha $^{-1}$ año $^{-1}$ en densidades de plantación superiores a las 50000 plantas ha $^{-1}$. La producción de biomasa fresca y seca, depende de una gran diversidad de factores, como el material genético utilizado, condiciones de manejo y ambientales, densidad de población, entre otros (González et al. 2018).

\section{CONCLUSIONES}

El efecto del contenido de humedad en el crecimiento, desarrollo y productividad del nopal, depende de la variedad utilizada. Las variedades Narro y Escobar fueron las de mejor respuesta en producción de bioamasa fresca y seca en contenidos de humedad del suelo en un rango aproximado a capacidad de campo (muy favorable), teniendo como principal componente de rendimiento la capacidad reproductiva de cladodios por planta. El rendimiento estimado de biomasa fresca fue de 10.1 a $11.8 \mathrm{tha}^{-1} \mathrm{y}$ de 0.998 a $1.156 \mathrm{t} \mathrm{ha}^{-1}$ de biomas seca. Con base al contenido relativo de agua (CRA) como indicador de tolerancia al estrés hídrico las variedades Chapingo, Narro y Escobar mantienen valores similares de producción de biomasa fresca y seca en condiciones hídricas de regualares a deficientes, por lo que, en condiciones de sequía, cualquiera de ellas puede ser utilizadas en términos de producción de forraje. El contenido de clorofila no es afectado en ninguna de las variedades de nopal.

\section{LITERATURA CITADA}

Aguilar BG, Peña VCB (2006) Alteraciones fisiológicas provocadas por sequía en nopal (Opuntia ficus-indica). Revista Fitotecnia Mexicana 29: 231-127. 
Alessio S, Brunella M, Paololnglese, Riccardo-Lo B (2016) Cladode growth dynamics in Opuntia ficus-indica under drought. Environmental and Experimental Botany 122: 158-167.

Aranda I, Ramírez-Valiente JA, Rodríguez-Calcerrada J (2014) Características funcionales que influyen en la respuesta a la sequía de las especies del género Quercus: variación inter-e intra-específica. Ecosistemas 23: 27-36.

Argentel L, González LM, Ávila C, Aguilera R (2006) Comportamiento del contenido relativo de agua y la concentración de pigmentos fotosintéticos de variedades de trigo cultivadas en condiciones de salinidad. Cultivos Tropicales 27: 49-53.

Azpilcueta-Pérez ME, Pedroza-Sandoval A, Trejo-Calzada R, Sánchez-Cohen I, Jacobo-Salcedo Ma. Del R (2018) Chemical residuality in maize (Zea mays L.) fields irrigated with deep well water. Revista Ecosistemas y Recursos Agropecuarios 5: 111-117.

Becerril AG, Peña VCB (2006) Alteraciones fisiológicas provocadas por sequía en nopal (Opuntia ficus-indica). Revista Fitotecnia Mexicana 29: 231-237.

Casierra-Posada F, Ávila-León OF y Riascos-Ortíz DH (2013) Cambios diarios del contenido de pigmentos fotosintéticos en hojas de caléndula bajo sol y sombra. Temas Agrarios 17: 60-71.

CONAGUA (2010) Comisión Nacional del Agua. Estadísticas agrícolas de los distritos de riego. Año agrícola 2008-2009. Secretaría de Medio Ambiente y Recursos Naturales. México. 323p.

FAO (2013) Tecnologías para el uso sostenible del agua. Oficina Subregional de FAO para Mesoamérica. Edificio 238, Ciudad del Saber, Clayton, Panamá. https://www.fao.org. Fecha de consulta: 7 de septiembre de 2020.

FAO-ICARDA (2018) Ecología del cultivo, manejo y usos del nopal. En: Inglese P, Mondragon JC, Ali Nefzaoui, ICARDA Edits). Organización de las Naciones Unidas para la Alimentación y la Agricultura y Centro Internacional de Investigaciones Agrícolas en Zonas Áridas, Roma. 229p. http://www.fao.org/3/i7628es/l7628ES. pdf. Fecha de consulta 5 de julio de 2021.

Florido BM, Bao FL (2014) Tolerancia a estrés por déficit hídrico en tomate (Solanum lycopersicum L.). Cultivos Tropicales 35: 70-88.

Flores-Hernández A, Macías RFJ, Esparza-Ibarra EL, Quiñones-Zaldivar A, Murillo-Amador M, García- Hernández J, Rueda-Puente EO (2019) Evaluación del nopal forrajero (Opuntia spp) en el Norte de México como opción para su enriquecimiento proteico. Sociedad Mexicana de Agricultura Sostenible, A.C. México. pp: 1474-1489.

Ferreira CA, Ferreira RLC, Santos DC, Santos MVF, Silva JAA, Lira MA, Molica SG (2003) Utilização de técnicas multivariadas na avaliação da divergência genética entre clones de palma forrageira (Opuntia fícus indica Mill). Revista Brasileira de Zootecnia 32: 1560-1568.

Feugang JM, Konarski P, Zou D, Stintzing FC, Zou C (2006) Nutritional and medicinal use of cactus pear (Opuntia spp.) cladodes and fruits. Frontiers in Bioscience 11: 2574-2589.

González AD, Álvarez HU, Lima OR (2018) Acumulación de biomasa fresca y materia seca por planta en el cultivo intercalado caupí - sorgo. Centro Agrícola 45: 77-82.

Jiménez-Galindo JC, Acosta-Gallegos JA (2013) Rendimiento de frijol común (Phaseolus vulgaris L.) y Tépari (Phaseolus acutifolius A. Gray) bajo el método riego-sequía en Chihuahua. Revista Mexicana de Ciencias Agrícolas 4: 557-567

Kramer PJ (1983) Water relation of plants. New York Academic Press. USA. 475p. 
López-Collado CJ, Malpica-Vázquez A, López -Collado J, García-Pérez E, Sánchez A (2013) Crecimiento de Opuntia ficus-indica (L.) Mill. en la zona central de Veracruz. Revista Mexicana de Ciencias Agrícolas 4: 1005-1014.

Medina GG, Díaz PG López HJ, Ruíz CJA, Marín SM 2005 Estadísticas climatológicas básicas del estado de Durango. (Periodo 1961 - 2003). Libro Técnico N 1. Campo Experimental Valle del Guadiana. CIRNOCINIFAP 224p.

Meléndez L, Hernández A, Fernández S (2006) Efecto de la fertilización foliar y edáfica sobre el crecimiento de plantas de maíz sometidas a exceso de humedad en el suelo. Bioagro 18: 107-114.

Montemayor-Trejo JA, Suárez-González E, Munguía-López JP, Segura-Castruita MA, Mendoza-Villarreal R, WooReza JL (2018) Acolchados plásticos para la producción de maíz (Zea mays L.) forrajero en la Comarca Lagunera. Revista Mexicana de Ciencias Agrícolas 9: 4107-4115.

Pedroza SA, Durán BS (2005) Efecto del acolchado plástico, fertilización nitrogenada y composta orgánica en el crecimiento y desarrollo de sábila Aloe barbadensis Miller, con riego por goteo presurizado. Revista Chapingo Serie Zonas Áridas 4: 1-7.

Pedroza SA, Ríos FJL, Torres MM, Cantú BJE, Piceno SC y Yáñez ChLG (2014) Eficiencia del agua de riego en la producción de maíz forrajero (Zea mays L.) y alfalfa (Medicago sativa): Impacto social y económico. Terra Latinoamericana 32: 231-239.

Pedroza SA (1995) El déficit hídrico en las plantas. Principios y técnicas de manejo. Unidad Regional Universitaria de Zonas Áridas-Universidad Autónoma Chapingo. Bermejillo, Dgo, México. 162p.

Richards LA (1948) Porous plate apparatus for measuring moisture retention and transmission by soil. Soil Science 66: 105-110.

Ruiz-Espinoza FH, Alvarado-Mendoza JF, Murillo-Amador B, García-Hernández JL, Pargas-Lara R, DuarteOsuna DO, Fenech-Larios L (2008) Rendimiento y crecimiento de nopalitos de cultivares de nopal (Opuntia ficus-indica) bajo diferentes densidades de plantación. Journal of the Professional Association for Cactus Development 10: 22-35

Sánchez H, Ochoa G, Alfaro R, Peña P (2018) Evaluación de la capacidad forrajera de Opuntia ficus. Manglar 14: 115-124.

SAGARPA (2015) Estudio de factibilidad para el establecimiento de cultivo de nopal (Opuntia) en tierras ociosas en los estados de Aguascalientes, San Luis Potosí, Guanajuato y Zacatecas con fines alimenticios, energéticos y ambientales. Informe detallado. SAGARPA-Universidad Autónoma de Zacatecas. México. 92p.

SIAP (2019) Servicio de Información Agroalimentaria y Pesquera, Información Ganadera. https://www.gob.mx/ siap/documentos/poblacion-ganadera-136762. Fecha de consulta: 4 de enero de 2021.

Torres-Ponce RL, Morales-Corral D, Ballinas-Casarrubias MDL, Nevárez-Moorillón GV (2015) El nopal: planta del semidesierto con aplicaciones en farmacia, alimentos y nutrición animal. Revista Mexicana de Ciencias Agrícolas 6: 1129-1142.

Trujillo ME, Méndez JR, Hossne AJ, Parra FJ (2010) Efecto de la humedad y compactación de un Ultisol de la sabana del estado Monagas sobre la concentración de clorofila y carotenoides, lavado de electrolitos y contenido relativo de agua en plantas de soya. Acta Universitaria 20: 18-30. 